\title{
Young adult mental health and functional outcomes among individuals with remitted, persistent and late-onset ADHD
}

\author{
Jessica C. Agnew-Blais, Guilherme V. Polanczyk, Andrea Danese, Jasmin Wertz, Terrie E. Moffitt and \\ Louise Arseneault
}

\section{Background}

Attention-deficit hyperactivity disorder (ADHD) is associated with mental health problems and functional impairment across many domains. However, how the longitudinal course of ADHD affects later functioning remains unclear.

\section{Aims}

We aimed to disentangle how ADHD developmental patterns are associated with young adult functioning.

\section{Method}

The Environmental Risk (E-Risk) Longitudinal Twin Study is a population-based cohort of 2232 twins born in England and Wales in 1994-1995. We assessed ADHD in childhood at ages 5 7,10 and 12 years and in young adulthood at age 18 years. We examined three developmental patterns of ADHD from childhood to young adulthood - remitted, persistent and late-onset ADHD - and compared these groups with one another and with non-ADHD controls on functioning at age 18 years. We additionally tested whether group differences were attributable to childhood IQ, childhood conduct disorder or familial factors shared between twins.

\section{Results}

Compared with individuals without ADHD, those with remitted ADHD showed poorer physical health and socioeconomic outcomes in young adulthood. Individuals with persistent or lateonset ADHD showed poorer functioning across all domains, including mental health, substance misuse, psychosocial, physical health and socioeconomic outcomes. Overall, these associations were not explained by childhood IQ, childhood conduct disorder or shared familial factors.

\section{Conclusions}

Long-term associations of childhood ADHD with adverse physical health and socioeconomic outcomes underscore the need for early intervention. Young adult ADHD showed stronger associations with poorer mental health, substance misuse and psychosocial outcomes, emphasising the importance of identifying and treating adults with ADHD.

\section{Declaration of interest}

None.

\section{Keywords}

ADHD; Iongitudinal; outcomes.

\section{Copyright and usage}

(c) The Royal College of Psychiatrists 2018
Children with attention-deficit hyperactivity disorder (ADHD) are at increased risk for a wide variety of adverse outcomes in adulthood, including mental health problems, substance misuse disorders and lower educational attainment. ${ }^{1}$ Adults with ADHD also exhibit poor functioning, such as higher rates of anxiety and depression, divorce, unemployment and criminal convictions. ${ }^{2,3}$ A metaanalysis of studies of children with ADHD found that about 15\% will continue to meet criteria for the disorder in adulthood. ${ }^{4}$ Although many studies have documented poor adult outcomes among children with ADHD, fewer have distinguished between remitted and persistent ADHD groups, thus whether developmental patterns of ADHD have an effect on adult functioning remains unclear. Children with ADHD may fare more poorly in adulthood because childhood problems set them on a course for poorer outcomes in later life. Alternatively, it could be that poor adult functioning is largely attributable to challenges of coping with current ADHD, with adverse outcomes concentrated among those for whom ADHD persists into adulthood. Furthermore, recent population-based research has identified a third developmental pattern of ADHD, in which adults with ADHD do not meet diagnostic criteria for the disorder in childhood. ${ }^{5-7}$ These studies found late-onset ADHD accounted for $67-90 \%$ of adults with ADHD. However, many questions remain regarding late-onset $\mathrm{ADHD}$, including the role substance misuse disorders may play in later-emerging ADHD symptoms. ${ }^{8}$ Although those with late-onset ADHD state their symptoms interfere with their lives, ${ }^{5,7}$ it is unclear the extent to which functioning may be adversely affected compared with those with childhood-onset ADHD and those without ADHD. The aim of our study was to disentangle how patterns of ADHD across development, specifically ADHD remission, persistence and late-onset, are associated with young adult functioning. If those with remitted ADHD show poorer functioning in young adulthood, this would suggest that childhood ADHD may exert a long-term effect on functioning. Alternatively, if poor functioning is concentrated among those with young adult ADHD (persistent or late-onset), this would suggest that ADHD may exert a concurrent effect on functioning. By taking a developmental approach to systematically examining outcomes among remitted, persistent and late-onset developmental patterns of ADHD in a longitudinal population-based cohort, we can clarify how childhood and adult ADHD affect young adult functioning to inform the nature and timing of interventions.

\section{Method}

\section{Study cohort}

Participants were members of the Environmental Risk (E-Risk) Longitudinal Twin Study, a birth cohort of 2232 British children drawn from a larger birth register of twins born in England and Wales in 1994-1995. ${ }^{9}$ Full details about the sample are reported elsewhere. ${ }^{10}$ The E-Risk sample was constructed in 1999-2000 when 1116 families (93\% of those eligible to participate in the study) with 5-year-old twins of the same gender participated in 
home visit assessments. This sample comprised 56\% monozygotic (MZ) and 44\% dizygotic (DZ) twin pairs; gender was evenly distributed within zygosity ( $49 \%$ boys). Families were recruited to represent the UK population with new-born children in the 1990s on the basis of residential location throughout England and Wales and mother's age; teenaged mothers with twins were overselected to replace high-risk families who were selectively lost to the register through non-response. Older mothers having twins via assisted reproduction were underselected to avoid an excess of well-educated older mothers. At follow-up, the study sample represented the full range of socioeconomic conditions in the UK, as reflected in the families' distribution on a neighbourhood-level socioeconomic index (A Classification Of Residential Neighbourhoods (ACORN), developed by CACI Inc. for commercial use); ${ }^{11}$ specifically, E-Risk families' ACORN distribution matched that of households nationwide.

Follow-up home visits were conducted when the children were aged 7 (98\% participation), 10 (96\%), 12 (96\%) and 18 years (93\%). Home visits at ages 5-12 years included assessments with participants and their mother; we conducted full interviews with participants only at age 18 years $(n=2066)$. There were no differences between those who did and did not take part at age 18 years on socioeconomic status when the cohort was initially defined $\left(\chi^{2}=\right.$ $0.86, P=0.65)$, IQ at age 5 years $(t=0.98, P=0.33)$, internalising or externalising problems $(t=0.40, P=0.69$ and $t=0.41, P=0.68)$ or rates of childhood $\mathrm{ADHD}$ at ages $5,7,10$ or 12 years $\left(\chi^{2}=\right.$ 2.12, $P=0.71$ ). With parents' permission, questionnaires were mailed to the children's teachers, who returned questionnaires for $94 \%$ of children at age 5 years, $93 \%$ of those followed up at age 7 years, $90.1 \%$ at age 10 years and $83 \%$ at age 12 years. The Joint South London and Maudsley and the Institute of Psychiatry Research Ethics Committee approved each phase of the study (ethics committee approval: \#1997/122). Parents gave written informed consent and twins gave assent between 5 and 12 years, and then written informed consent at age 18 years.

\section{Childhood ADHD diagnoses}

We ascertained childhood ADHD diagnoses on the basis of mother and teacher reports of 18 symptoms of inattention and hyperactivity-impulsivity derived from DSM-IV diagnostic criteria and the Rutter Child Scales. ${ }^{12}$ Participants had to have six or more symptoms reported by mothers or teachers in the past 6 months, and the other informant must have endorsed at least two symptoms. We considered participants to have a diagnosis of childhood ADHD if they met criteria at age $5,7,10$ or 12 years. At age 5 years, $6.8 \%(n=131)$ of participants met the criteria for ADHD, $5.4 \%(n=102)$ met the criteria at age 7 years, $3.4 \%(n=65)$ met the criteria at age 10 years and $3.4 \%(n=64)$ met the criteria at age 12 years. In childhood, $0.8 \%$ of the study population $(n=17)$ were taking ADHD medication based on maternal report; rates of medication use were similar to those in the UK overall. ${ }^{13}$

\section{ADHD diagnosis at age 18 years}

We ascertained ADHD diagnosis at age 18 years based on private structured interviews with participants regarding 18 symptoms of inattention and hyperactivity-impulsivity according to DSM- 5 criteria. ${ }^{5,14}$ Participants had to endorse five or more inattentive and/or five or more hyperactivity-impulsivity symptoms to be diagnosed; we also required that symptoms interfere with individual's life at 'home, or with family and friends' and at 'school or work', thereby meeting impairment and pervasiveness criteria. The requirement of symptom onset before age 12 years was met if parents or teachers reported more than two ADHD symptoms at any childhood assessment. A total of $8.1 \%$ of participants $(n=$
166) met criteria for ADHD at age 18 years. Analyses were restricted to those individuals with information on childhood and young adult ADHD $(n=2040)$.

Participants additionally nominated two people who knew them well to provide information about the participant on questionnaires, including eight items related to ADHD. In total, $99.3 \%$ of participants had information from co-informants, including $81.1 \%$ from both a parent and co-twin, $17.2 \%$ from a co-twin only and $1.7 \%$ from a parent only.

\section{Remitted, persistent and late-onset ADHD groups}

We identified three groups of individuals with ADHD across childhood and young adulthood: $9.5 \%$ of participants $(n=193)$ showed remitted ADHD (met diagnostic criteria in childhood but not at age 18 years), $2.6 \%(n=54)$ showed persistent ADHD (met diagnostic criteria in childhood and age 18 years $)$ and $5.5 \%(n=112)$ showed late-onset ADHD (did not meet diagnostic criteria in childhood but did at age 18 years). At age 18 years, $0.6 \%(n=13)$ of the study population reported taking ADHD medication: $15.4 \%(n=8)$ of those with persistent ADHD and $4.5 \%(n=5)$ of those with lateonset ADHD. A total of $82.4 \%$ participants $(n=1681)$ did not meet the criteria for ADHD in childhood or adulthood.

\section{Young adult outcomes}

\section{Mental health}

Participants were interviewed for the presence of depressive disorder, generalised anxiety disorder and conduct disorder, according to DSM-IV criteria. ${ }^{15}$ Assessments were conducted in face-to-face interviews using the Diagnostic Interview Schedule (DIS), ${ }^{15}$ with the exception of conduct disorder, which was included in a computer-assisted module. Suicide attempt was defined as any selfreported suicide attempt between ages 12 and 18 years. Self-harm was defined as positive response to the question 'Have you ever tried to hurt yourself to cope with emotional stress or pain?'.

\section{Substance misuse}

Alcohol and cannabis dependence over the previous 12 months were evaluated with DSM-IV criteria during face-to-face interviews, using the DIS. ${ }^{15}$ Other illicit substance use included non-prescription use of stimulants, sedatives, cocaine/crack, painkillers, street opiates, club drugs, hallucinogens, inhalants and 'other drugs'.

\section{Psychosocial outcomes}

Life satisfaction was assessed by the Satisfaction with Life Scale ${ }^{16}$ and social isolation via the Multidimensional Scale of Perceived Social Support. ${ }^{16}$ Problematic technology use was assessed with the Compulsive Internet Use Scale, ${ }^{17}$ adapted to include use of the internet, email, social networking sites and tools, mobile phones and text messaging to update the measure to reflect the current nature of online activities and communication.

\section{Physical health}

Body mass index (BMI) was calculated by dividing weight in kilograms by height in metres squared; obesity was defined as $\mathrm{BMI} \geq 30$. Daily cigarette smoking was assessed by asking the participant if they had ever smoked a cigarette, followed by if and when they began smoking every day; current daily smokers were participants who endorsed daily smoking within the past year. Participants self-reported whether they had visited an accident and emergency department in the past year. 


\section{Socioeconomic outcomes}

Low educational attainment was assessed by whether participants failed to obtain or scored low (grades D-G) on their General Certificate of Secondary Education (GCSE). GCSEs are a standardised examination taken at the end of compulsory education at age 16 years. Individuals were considered to be not in education, employment or training (NEET) if they reported that they were not studying, working in paid employment or pursuing a vocational qualification or apprenticeship training (for reasons other than being on holiday or being a parent). Criminal cautions and convictions were assessed through the UK Police National Computer records searched by the UK Ministry of Justice, and included participants cautioned or convicted in the UK up to age 19 years.

\section{Childhood confounders}

All regression models were adjusted for gender and childhood socioeconomic status. Childhood socioeconomic status reflected a composite of parental income, education and occupation. Individuals with ADHD are more likely to have lower IQ and comorbid conduct disorder, ${ }^{12,18}$ and it is possible that these factors, rather than ADHD, lead to poor outcomes. Childhood IQ was assessed at age 5 years by two subtests of the Wechsler Preschool and Primary Scale of Intelligence - Revised. ${ }^{19}$ Childhood conduct disorder was assessed by DSM-IV criteria with the Achenbach family of instruments and additional items covering aggressive and nonaggressive conduct problems, deceitfulness or theft, and rule violations as reported by mothers and teachers from ages 5 to 12 years. ${ }^{20}$

\section{Statistical analyses}

To understand how different developmental patterns of ADHD were associated with young adult functioning, we compared groups with remitted, persistent and late-onset ADHD by age 18 years with individuals who had never had ADHD. We estimated effect sizes and significance of differences between groups by logistic and linear regressions, adjusting for gender and childhood socioeconomic status. We further adjusted for childhood conduct disorder and IQ in models predicting young adult outcomes. To assess whether findings could be artefacts from self-report of ADHD symptoms at age 18 years, we also conducted sensitivity analyses replacing ADHD diagnosis based on self-report with coinformant-rated ADHD symptoms at age 18 years. All of the above analyses adjusted for the non-independence of twin observations by the sandwich variance estimator in Stata version $14 .^{21}$ Aspects of the home environment and genetic make-up influence both the risk for ADHD and for poor outcomes in young adulthood; therefore we compared functioning between co-twins discordant for ADHD. MZ twins with ADHD (either in childhood and/or young adulthood, $n=91$ twin pairs, $55.5 \%$ boys) were compared with their co-twin who had never had ADHD by logistic regression.

\section{Results}

Young adult $\mathrm{ADHD}$ appeared to be a more salient risk factor for poor mental health than childhood ADHD per se. Overall, individuals with ADHD only in childhood (remitted ADHD) did not have poorer mental health at age 18 years compared with those who had never had ADHD, with the exception of conduct disorder (Table 1). However, those with persistent and late-onset ADHD had more depression, anxiety, conduct disorder and suicide/self-harm compared with those who had never had ADHD. Persistent and late-onset $\mathrm{ADHD}$ groups appeared to be equally impaired for mental health, as indicated by comparable effect sizes for these groups.
Similarly, those with persistent and late-onset ADHD showed elevated risk for cannabis dependence and illicit drug use at age 18 years compared with individuals who had never had ADHD, whereas those with remitted ADHD did not. Again, effect sizes for the late-onset group were similar to those of the persistent group. Only the late-onset $\mathrm{ADHD}$ group showed increased alcohol dependence compared with those without ADHD.

Psychosocial outcomes showed a similar pattern of findings, in which those with remitted ADHD had minimal impairment compared with those who had never had ADHD, whereas persistent and late-onset ADHD groups showed poorer functioning. The remitted ADHD group showed only lower life satisfaction compared with those without ADHD, whereas the persistent group had lower life satisfaction and more problematic technology use. The late-onset ADHD group showed poorer outcomes across each psychosocial outcome compared with those who had never had ADHD, with a similar effect size as the persistent ADHD group.

The pattern of findings was mixed for physical health outcomes. Both remitted and persistent groups showed greater obesity risk compared with those who had never had ADHD, whereas the late-onset group did not, suggesting a long-term effect of childhood ADHD. The risk of daily cigarette smoking was elevated in all ADHD groups, with similar effect sizes across all groups. For visits to emergency department, however, those with remitted ADHD showed no increased risk whereas both the persistent and late-onset groups showed an increased risk.

All ADHD groups showed poorer socioeconomic outcomes at age 18 years. Risk for low educational attainment was further elevated among individuals with persistent ADHD compared with those with remitted $\mathrm{ADHD}$ and those with late-onset $\mathrm{ADHD}$. Remitted, persistent and late-onset ADHD groups also showed higher risk for NEET and for criminal cautions/convictions, suggesting the presence of both long-term and concurrent associations of ADHD with these outcomes.

\section{Adjusting for childhood IQ and conduct disorder}

Overall, further adjustment for childhood IQ and conduct disorder did not account for poorer functioning at age 18 years among individuals with remitted, persistent or late-onset ADHD (Table 2). Exceptions to this were concentrated among physical health and socioeconomic outcomes; for example, after controlling for childhood IQ and conduct disorder, the risk of daily cigarette smoking was reduced, especially among the persistent ADHD group, and the risk of criminal cautions/convictions was lowered to non-significance among the persistent and late-onset ADHD groups.

\section{Accounting for familial and genetic influences}

Compared with their unaffected co-twin, twins with ADHD in childhood or adulthood were more likely to experience young adult depression, anxiety, suicide/self-harm, daily smoking, low educational attainment, lower life satisfaction and more problematic technology use (Fig. 1). Therefore, these poor outcomes among participants with ADHD were not attributable to risk factors shared with their MZ co-twin, including genetic or family environmental factors such as parental psychopathology or chaotic home environment.

\section{Sensitivity analyses by co-informant report of ADHD symptoms at age 18 years}

Results were unchanged for all outcomes based on co-informantrated ADHD symptoms (Table 3). For mental health, substance misuse and psychosocial outcomes, co-informant-rated symptoms in young adulthood were a more salient risk factor compared 
Table 2 Mental health and functional outcomes at age 18 years among participants with remitted, persistent and late-onset ADHD compared with those who had never had ADHD, further adjusting for childhood IQ and conduct disorder

\begin{tabular}{|c|c|c|c|c|c|c|}
\hline \multirow[t]{2}{*}{ Young adult outcome } & \multicolumn{2}{|c|}{ Remitted ADHD } & \multicolumn{2}{|c|}{ Persistent ADHD } & \multicolumn{2}{|c|}{ Late-onset ADHD } \\
\hline & Odds ratio $(95 \% \mathrm{Cl})$ & $P$-value & Odds ratio $(95 \% \mathrm{Cl})$ & $P$-value & Odds ratio $(95 \% \mathrm{Cl})$ & $P$-value \\
\hline \multicolumn{7}{|l|}{ Mental health } \\
\hline Depression & $1.27(0.84-1.92)$ & 0.265 & $2.68(1.40-5.13)$ & 0.003 & $3.37(2.24-5.06)$ & $<0.001$ \\
\hline Anxiety & $1.10(0.50-2.41)$ & 0.811 & 6.47 (3.09-13.53) & $<0.001$ & $2.95(1.72-5.04)$ & $<0.001$ \\
\hline Conduct disorder & $1.03(0.66-1.58)$ & 0.911 & $2.15(1.03-4.49)$ & 0.041 & $3.48(2.20-5.49)$ & $<0.001$ \\
\hline Suicide/self-harm & $0.92(0.58-1.48)$ & 0.740 & $2.38(1.13-5.00)$ & 0.023 & $2.18(1.39-3.43)$ & 0.001 \\
\hline \multicolumn{7}{|l|}{ Substance misuse } \\
\hline Cannabis dependence & $0.82(0.41-1.65)$ & 0.576 & $2.65(1.05-6.72)$ & 0.040 & $3.02(1.46-6.25)$ & 0.003 \\
\hline Illicit drug use & $1.01(0.66-1.55)$ & 0.971 & $1.63(0.82-3.25)$ & 0.162 & $2.13(1.32-3.43)$ & 0.002 \\
\hline Alcohol dependence & $1.34(0.85-2.12)$ & 0.206 & $1.01(0.39-2.61)$ & 0.988 & $3.70(2.38-5.76)$ & $<0.001$ \\
\hline Psychosocial & $\beta(95 \% \mathrm{Cl})$ & $P$-value & $\beta(95 \% \mathrm{Cl})$ & $P$-value & $\beta(95 \% \mathrm{Cl})$ & $P$-value \\
\hline Life satisfaction & $-0.03(-0.07$ to 0.02$)$ & 0.251 & $-0.08(-0.14$ to -0.01$)$ & 0.021 & $-0.14(-0.19$ to -0.08$)$ & $<0.001$ \\
\hline Social isolation & $-0.01(-0.07$ to 0.04$)$ & 0.653 & 0.03 (-0.04 to 0.09) & 0.413 & $0.06(0.01-0.11)$ & 0.025 \\
\hline Problematic technology use & $0.03(-0.03$ to 0.08$)$ & 0.337 & $0.14(0.07-0.21)$ & $<0.001$ & $0.23(0.17-0.28)$ & $<0.001$ \\
\hline \multicolumn{7}{|l|}{ Physical health } \\
\hline Obesity & $1.63(0.91-2.93)$ & 0.098 & $2.18(0.91-5.23)$ & 0.080 & $1.19(0.63-2.26)$ & 0.597 \\
\hline Daily cigarette smoking & $1.56(1.07-2.28)$ & 0.021 & $1.53(0.79-2.95)$ & 0.203 & $2.24(1.45-3.47)$ & $<0.001$ \\
\hline Emergency department visit & $1.10(0.75-1.63)$ & 0.616 & $1.55(0.84-2.87)$ & 0.164 & $1.69(1.10-2.58)$ & 0.016 \\
\hline \multicolumn{7}{|l|}{ Socioeconomic } \\
\hline Low educational attainment & $3.39(2.27-5.07)$ & $<0.001$ & $5.84(3.12-10.91)$ & $<0.001$ & $1.98(1.20-3.25)$ & 0.007 \\
\hline NEET status & $2.05(1.32-3.18)$ & 0.001 & $2.76(1.33-5.73)$ & 0.006 & $1.57(0.87-2.86)$ & 0.136 \\
\hline Criminal cautions/convictions & $1.65(1.08-2.50)$ & 0.020 & $1.51(0.75-3.06)$ & 0.250 & $1.42(0.74-2.73)$ & 0.289 \\
\hline
\end{tabular}

with childhood ADHD diagnosis. Both co-informant-rated ADHD symptoms and childhood ADHD were associated with risk of daily cigarette smoking, low educational attainment status and not being in education, employment or training, mirroring results from selfreported data. Additionally, as with self-reported ADHD, coinformant-reported ADHD symptoms in young adulthood were not associated with increased obesity risk.

\section{Supplemental analyses}

In supplemental analyses, we ruled out the possibility that the associations between young adult ADHD and functioning at age 18 years were attributable to individuals with persistent ADHD having more frequent diagnoses of ADHD across childhood (supplementary Table 1 available at https://doi.org/10.1192/bjp. 2018.97), or to subthreshold childhood ADHD symptoms among those with late-onset ADHD (supplementary Table 2). Additionally, because recent research has suggested late-onset ADHD may be accounted for by substance misuse disorders, ${ }^{8}$ we examined findings excluding participants with alcohol dependence, marijuana dependence or illicit drug use (supplementary Table 3). Overall results were similar, however, daily cigarette smoking and visits to the emergency department were no longer significantly elevated among both the persistent and late-onset ADHD groups.

\section{Discussion}

Children and adults with ADHD are more likely to experience a range of negative outcomes in adulthood, which are distressing for the affected individual, concerning for family members and costly for society. ${ }^{22}$ Poor mental health and functioning in the transition to adulthood can have far-reaching consequences into later life: young adult outcomes such as depression, low educational attainment, smoking and obesity may have a major effect on quality of life into mid-life and beyond, and can even lead to premature mortality. In this study, we found that the developmental pattern of ADHD from childhood into young adulthood influences functional outcomes, and this effect varies by outcome domain. Those with remitted ADHD showed poor socioeconomic and physical health outcomes in young adulthood, suggesting that childhood ADHD has a long-term association with these domains even if an individual no longer meets criteria for the disorder. Conversely, those with young adult ADHD (both persistent and late-onset) showed adverse socioeconomic and physical health outcomes, as well as poorer mental health, substance misuse and psychosocial outcomes.

\section{Long-term effects of childhood ADHD}

Childhood ADHD appears to maintain a long-term effect on later functioning for those outcomes more overtly cumulative in nature. For example, educational attainment builds on past performance, and it may be difficult to rebound from prior school failures even if ADHD symptoms abate. Our finding of lower educational attainment among those with remitted and persistent ADHD is consistent with another population-based study that showed participants with 'high' as well as 'declining' inattention symptom trajectories both had lower rates of graduating high school compared with those with consistently 'low' symptom levels. ${ }^{23}$

Childhood ADHD was also associated with a more than $70 \%$ increased risk for obesity at age 18 years, even when the disorder remitted by young adulthood. Studies have found children with ADHD are more likely to be obese compared with those without ADHD. ${ }^{24}$ One possibility for the long-term effect is that higher BMI, once established in childhood, is relatively intractable such that later changes to ADHD symptom levels may not affect BMI. Additionally, recent research has found overlap in genetic risk for ADHD and obesity; ${ }^{25}$ indeed, our twin analyses did not show significant differences in obesity between ADHD-discordant MZ twins and so we cannot rule out that shared genetics may increase risk for both ADHD and obesity.

\section{Concurrent effects of young adult ADHD}

Although remission of ADHD appeared to be associated with a corresponding normalisation of risk for most mental health problems, substance misuse and psychosocial problems, ADHD in young adulthood was associated with poor functioning across 
(a)

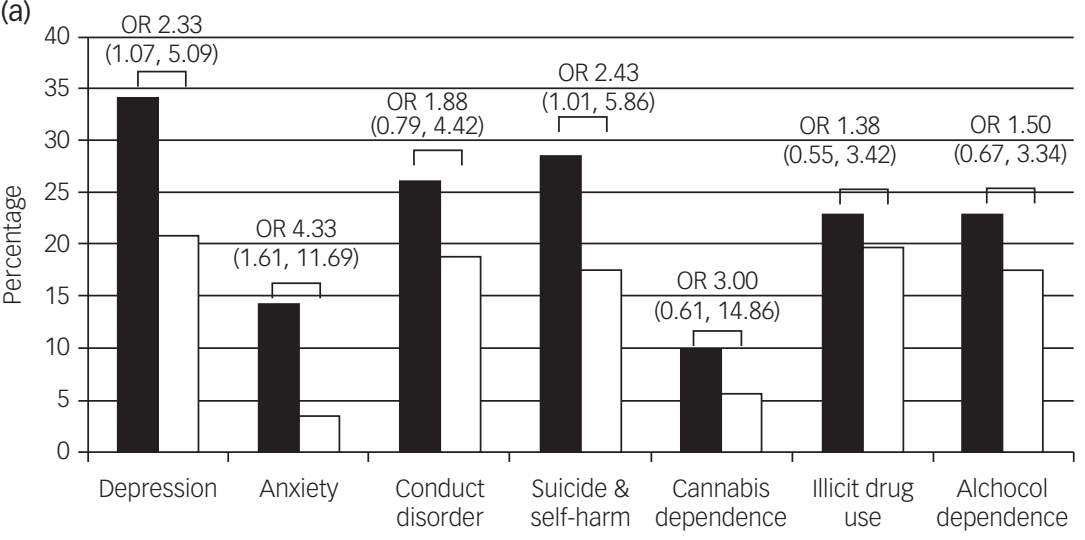

ADHD twin $\square$ Unaffected co-twin
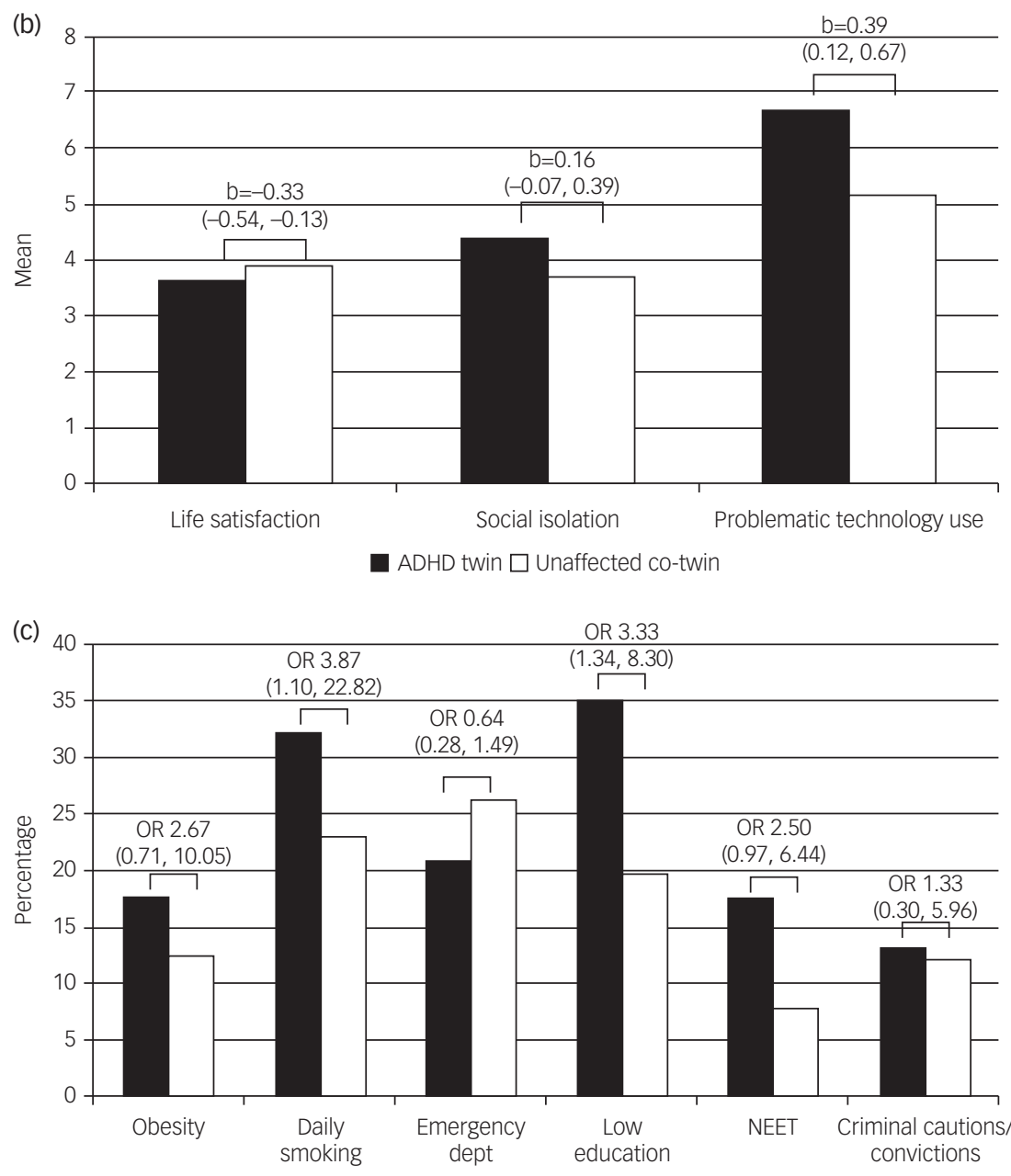

Fig. 1 Discordant monozygotic twin analyses ( $n=91$ twin pairs) comparing outcomes among twins with ADHD and their unaffected co-twin, with significant differences in bold. (a) Mental health and substance misuse. (b) Psychosocial functioning. (c) Physical health and socioeconomic outcomes.

$95 \% \mathrm{Cl}$ values have been indicated within parentheses.

ADHD, attention-deficit hyperactivity disorder; NEET, not in education, employment or training; OR, odds ratio.

each of these domains. More proximal pathways may link young adult ADHD with risk for other mental health problems; for example, individuals with young adult ADHD may have more interpersonal problems, failures at school or frustrations at work, which could lead to depression or anxiety. Additionally, the outcomes most affected by young adult ADHD may be those that more explicitly tap into current functioning; for example, problematic technology use or daily cigarette smoking may reflect ways in which individuals cope with their ADHD symptoms.

That individuals with persistent ADHD fared more poorly than those who remitted is consistent with recent studies in referred 


\section{Odds ratio}

$95 \% \mathrm{Cl}$

Mental health

Depression

Co-informant-rated ADHD symptoms

Childhood ADHD diagnosis

1.22

Anxiety

Co-informant-rated ADHD symptoms

Childhood ADHD diagnosis

Conduct disorder

Co-informant-rated ADHD symptoms

Childhood ADHD diagnosis

Suicide/self-harm

Co-informant-rated ADHD symptoms Childhood ADHD diagnosis

Substance misuse

Cannabis dependence

Co-informant-rated ADHD symptoms

Childhood ADHD diagnosis

Illicit drug use

Co-informant-rated ADHD symptoms

Childhood ADHD diagnosis

Alcohol dependence

Co-informant-rated ADHD symptoms

Childhood ADHD diagnosis

Psychosocial

Life satisfaction

Co-informant-rated ADHD symptoms

Childhood ADHD diagnosis

Social isolation

Co-informant-rated ADHD symptoms

Childhood ADHD diagnosis

Problematic technology use

Co-informant-rated ADHD symptoms Childhood ADHD diagnosis

Physical health

Obesity

Co-informant-rated ADHD symptoms

Childhood ADHD diagnosis

Daily cigarette smoking

Co-informant-rated ADHD symptoms

Childhood ADHD diagnosis

Emergency department visit

Co-informant-rated ADHD symptoms

Childhood ADHD diagnosis

Socioeconomic

Low educational attainment

Co-informant-rated ADHD symptoms

Childhood ADHD diagnosis

NEET status

Co-informant-rated ADHD symptoms

Childhood ADHD diagnosis

Criminal cautions/convictions

Co-informant-rated ADHD symptoms

Childhood ADHD diagnosis
1.25

1.12

1.53

1.30

1.21

1.24

1.03

1.32

0.94

1.24

0.95

1.18

1.03

b

$-0.09$

$-0.07$

0.33

0.06

0.34

0.77

1.07

1.80

1.35

1.44

1.11

1.16

1.20

4.65

1.23

2.39

1.20

1.97
(1.13 to 1.31 )

(0.86 to 1.82 )

(1.01 to 1.24 )

(0.88 to 2.64 )

(1.20 to 1.40$)$

(0.83 to 1.78 )

(1.15 to 1.35 )

(0.67 to 1.59 )

(1.19 to 1.48 )

(0.45 to 1.97 )

(1.15 to 1.35$)$

(0.63 to 1.42 )

(1.09 to 1.27 )

(0.67 to 1.58 )

$95 \% \mathrm{Cl}$

$(-0.13$ to -0.06$)$

$(-0.18$ to 0.04$)$

(0.16 to 0.50 )

$(-0.66$ to 0.76$)$

(0.07 to 1.47 )

$(-0.68$ to 0.08$)$

(0.96 to 1.19 )

(1.13 to 2.88 )

(1.25 to 1.46$)$

(1.02 to 2.03)

(1.02 to 1.19 )

(0.82 to 1.64)

(1.10 to 1.30 )

(3.28 to 6.60 )

(1.12 to 1.35 )

(1.60 to 3.57 )

(1.09 to 1.31)

(1.34 to 2.90 )
$P$-value

$<0.001$

0.239

0.028

0.130

$<0.001$

0.323

$<0.001$

0.890

$<0.001$

0.870

$<0.001$

0.785

$<0.001$

0.898

$P$-value

$<0.001$

0.234

$<0.001$

0.871

$<0.001$

0.030

0.215

0.013

$<0.001$

0.039

$\mathbf{0 . 0 1 2}$

0.400

$<0.001$

$<0.001$

$<0.001$

$<0.001$

$<0.001$

0.010

Significant values are in bold.

b, regression coefficient; ADHD, attention-deficit hyperactivity disorder; NEET, not in education, employment or training.

samples of ADHD children and adolescents which indicate that those with persistent ADHD have poorer mental health, higher risk of self-injurious behaviour and more substance misuse disorders. ${ }^{26-28}$ We have extended these findings in a population-based cohort and showed that poorer functioning among those with persistent ADHD was not accounted for by childhood lower IQ or conduct disorder, nor was it because those with persistent ADHD had more frequent ADHD diagnoses across childhood compared with those who remitted. Additionally, discordant twin analyses revealed that these associations were not attributable to childhood family environmental risk factors (e.g. parental psychopathology) or genes that increase risk for ADHD and other disorders because, despite sharing both a home environment and 100\% of their genes, the twins with ADHD had significantly poorer mental health and psychosocial functioning than the twins without ADHD.

We have also extended prior research to show that young adult $\mathrm{ADHD}$ is associated with poor functioning not only for those whose ADHD persisted from childhood, but also for those with late-onset ADHD. Functioning in the late-onset ADHD group was as impaired as the persistent group across several domains; for example, over one-quarter of both groups had attempted suicide or engaged in self-harm behaviour. Poor outcomes were not attributable to the late-onset ADHD group having lower socioeconomic status, IQ or more conduct disorder in childhood. 
Additionally, our supplemental analyses showed that poorer functioning in young adulthood in this group was not explained by subthreshold ADHD symptoms in childhood among those who later met criteria for late-onset ADHD. Furthermore, functioning was also poorer among those with late-onset ADHD who did not have a comorbid substance misuse problem. Exceptions to this were daily cigarette smoking and emergency department visits, for which risk was no longer significantly elevated compared with controls among the persistent and late-onset ADHD groups after excluding those with substance misuse, suggesting perhaps a specific pathway between young adult ADHD and these outcomes operating through substance misuse problems. Although much about late-onset ADHD remains to be explored, our finding that individuals with late-onset $\mathrm{ADHD}$ were as impaired as those with persistent ADHD in many domains in young adulthood points to the need for appropriate clinical attention for these individuals.

\section{Limitations}

Our study has several strengths, including multiple assessments of ADHD across childhood and into young adulthood in a population-based cohort with an over $90 \%$ retention rate. However, we need to consider our findings in light of some limitations. Although the temporal ordering of childhood ADHD and functioning at 18 years outcomes is clear, we are more limited in the causal inferences we can draw between young adult ADHD and functioning at 18 years, as they were assessed concurrently. Therefore, it is possible, for example, that lower educational attainment contributed to the persistence of $\mathrm{ADHD}$, rather than persistent $\mathrm{ADHD}$ affecting educational attainment. Additionally, we derived ADHD diagnoses at age 18 years from self-reported information only, rather than parental report. Concerns have been raised about individual's ability to report on their own ADHD symptoms. However, prior work in this cohort found co-informant report of ADHD symptoms at age 18 years to corroborate self-reports: those with self-reported late-onset ADHD have significantly more co-informant-rated ADHD symptoms than those without ADHD, and those with persistent ADHD are rated by co-informants as having more ADHD symptoms at age 18 years than those with remitted ADHD. ${ }^{5}$ Furthermore, recent research with a self-reported ADHD symptom scale found high specificity and positive predictive value compared with a diagnostic interview administered by clinicians. ${ }^{29}$ Additionally, in sensitivity analyses, our results regarding the salience of childhood versus young adult ADHD for later functioning did not change when using co-informant-rated ADHD symptoms at age 18 years. The E-Risk study sample does not include a follow-up assessment between the ages of 12 and 18 years, therefore we cannot determine exactly the age at which ADHD remitted or emerged if it did so in adolescence. Our sample comprised twins, thus results may not generalise to singletons; however, as reported previously, the prevalence of ADHD at each age in our cohort is well within ranges estimated in other samples. ${ }^{30}$ In addition, childhood ADHD was associated with previously identified risk factors, ${ }^{31}$ and our rate of $\mathrm{ADHD}$ persistence is similar to that found by a meta-analysis. ${ }^{4}$ Finally, we have followed participants only to the beginning of young adulthood and future research is needed to parse the long-term effects of childhood versus concurrent ADHD in later life, which could include additional outcomes such as longer-term employment issues, parenting behaviours and chronic disease.

\section{Conclusions and implications}

If $\mathrm{ADHD}$ resolves, children with $\mathrm{ADHD}$ are not destined to experience negative sequelae across all domains of functioning in young adulthood. However, the long-term effect of childhood ADHD on physical health and socioeconomic outcomes underscores the need for early intervention. For mental health, substance misuse and psychosocial outcomes, young adult ADHD showed a stronger association with functioning, emphasising the importance of identifying and treating young adult ADHD. The presence of significant impairments in the late-onset ADHD group suggests that individuals with this developmental pattern, who may not be captured with the current classification systems, require attention and possibly treatment.

Jessica C. Agnew-Blais, SCD, MRC Skills Development Fellow, Social, Genetic and Developmental Psychiatry, Institute of Psychiatry, Psychology and Neuroscience, King's College London, UK; Guilherme V. Polanczyk, MD, PhD, Assistant Professor,

Department of Psychiatry, University of São Paulo Medical School, Brazil; Andrea Danese, MD, PhD, Reader in Developmental Psychobioligy and Psychiatry, Consultant Child and Adolescent Psychiatrist, Social, Genetic and Developmental Psychiatry, Institute of Psychiatry, Psychology and Neuroscience, King's College London, UK, Department of Child and Adolescent Psychiatry, Institute of Psychiatry, Psychology and Neuroscience, King's College London, UK and National and Specialist Child Traumatic Stress and Anxiety Clinic, South London and Maudsley NHS Foundation Trust, UK; Jasmin Wertz, PhD, Postdoctoral Fellow, Social, Genetic and Developmental Psychiatry, Institute of Psychiatry, Psychology and Neuroscience, King's College London, UK and Department of Psychology and Neuroscience, Duke University, USA; Terrie E. Moffitt, PhD, Professor, Social, Genetic and Developmental Psychiatry, Institute of Psychiatry, Psychology and Neuroscience, King's College London, UK, Department of Psychology and Neuroscience, Duke University, USA and Department of Psychiatry and Behavioral Sciences, Duke University Medical Center, USA; Louise Arseneault, PhD, Professor and ESRC Mental Health Leadership Fellow, Social, Genetic and Developmental Psychiatry, Institute of Psychiatry, Psychology and Neuroscience, King's College London, UK

Correspondence: Louise Arseneault, Institute of Psychiatry, Psychology \& Neuroscience, King's College London, Box number P080, 16 De Crespigny Park, London SE5 8AF, UK. Email: louise.arseneault@kcl.ac.uk

First received 13 Jan 2018, final revision 18 Apr 2018, accepted 25 Apr 2018

\section{Supplementary material}

Supplementary material is available online at https://doi.org/10.1192/bjp.2018.97.

\section{Funding}

The E-Risk study is funded by the Medical Research Council (grant G1002190). Additional support was provided by the National Institute of Child Health and Human Development (grant HD077482) and by the Jacobs Foundation.

\section{Acknowledgements}

We are grateful to the study members and their families for their participation. We also thank the Avielle Foundation, $\mathrm{CACl}$, Inc., the UK Ministry of Justice and members of the E-Risk team for their dedication, hard work and insights. Finally, we thank Avshalom Caspi, PhD (Duke University and King's College London) and Sir Michael Rutter, MD, FRS (King's College London) for their involvement in establishing the E-Risk cohort. No compensation was received for this involvement. J.A.-B. is a Medical Research Council Skills Development Fellow. L.A. is the Economic and Social Research Council Mental Health Leadership Fellow.

\section{References}

1 Erskine HE, Noman RE, Ferrari AJ, Chan GCK, Copeland WE, Whiteford HA, et al. Long-term outcomes of attention-deficit/hyperactivity disorder and conduct disorder: a systematic review and meta-analyses. J Am Acad Child Adol Psychiatry 2016; 55(10): 841-50.

2 Kessler RC, Adler LA, Barkley R, Biederman J, Conners SCK, Faraone SV, et al. Patterns and predictors of attention-deficit/hyperactivity disorder persistence into adulthood: results from the national comorbidity survey replication. Biol Psychiatr 2005; 57(11): 1442-51.

3 Lichtenstein P, Halldner L, Zetterqvist K, Sjolander A, Serlachius E, Fazel S, et al. Medication for attention deficit-hyperactivity disorder and criminality. N Engl J Med 2012; 367(21): 2006-14.

4 Faraone SV, Biederman J. What is the prevalence of adult ADHD? Results from a population screen of 966 adults. J Atten Disord 2005; 9(2): 384-91.

5 Agnew-Blais JC, Polanczyk GV, Danese A, Wertz J, Moffitt TE, Arseneault L. Persistence, remission and emergence of ADHD in young adulthood: results from a longitudinal, prospective population-based cohort. JAMA Psychiatry 2016; 73(7): 713-20. 
6 Caye A, Rocha TBM, Anselmi L, Murray J, Menezes AMB, Barros F, et al. Attention-deficit/hyperactivity disorder trajectories from childhood to young adulthood: evidence from a birth cohort supporting a late-onset syndrome. JAMA Psychiatry 2016; 73(7): 705-12.

7 Moffitt TE, Houts R, Asherson P, Belsky DW, Corcoran DL, Hammerle M, et al. Is adult ADHD a childhood-onset neurodevelopmental disorder? Evidence from a four-decade longitudinal cohort study. Am J Psychiatry 2015; 172(10): 976-7.

8 Sibley MH, Rohde LA, Swanson JM, Hechtman LT, Molina BSG, Mitchell JT, et al. Late-onset ADHD reconsidered with comprehensive repeated assessments between ages 10 and 25. Am J PSychiatry 2018; 175(2): 140-9.

9 Trouton A, Spinath F, Plomin R. Twins Early Development Study (TEDS): a multivariate, longitudinal genetic investigation of language, cognition and behavior problems in childhood. Twin Res 2002; 5(5): 444-8.

10 Moffitt TE, E-Risk Study Team. Teen-aged mothers in contemporary Britain. J Child Adolesc Psychiatry 2002; 43(6): 727-42.

11 Odgers C, Caspi A, Russell M, Sampson R, Arseneault L, Moffitt TE. Supportive parenting mediates neighborhood socioeconomic disparities in children's antisocial behavior from ages 5 to 12. Devel Psychopathol 2012 24(3): 705-21.

12 Kuntsi J, Eley TC, Taylor A, Hughes C, Asherson P, Caspi A, et al. Co-occurrence of $A D H D$ and low IQ has genetic origins. Am J Med Genet $B$ Neuropsychiatr Genet 2004; 124B: 41-7.

13 McCarthy S, Wilton L, Murray ML, Hodgkins P, Asherson P, Wong IC. The epidemiology of pharmacologically treated attention deficit hyperactivity disorde (ADHD) in children, adolescents and adults in UK primary care. BMC Pediatrics 2012; 12(1): 78

14 American Psychiatric Association. Diagnostic and Statistical Manual of Mental Disorders: DSM-5. American Psychiatric Association, 2013.

15 Robins L, Cottler L, Bucholz K, Compton W. Diagnostic Interview Schedule for DSM-IV. Washington University School of Medicine, 1995.

16 Diener E, Emmons RA, Larsen RJ, Griffin S. The Satisfaction with Life Scale. J Pers Assess 1985; 49: 71-5.

17 Zimet G, Dahlem N, Zimet S, Farley G. The Multidimensional Scale of Perceived Social Support. J Pers Assess 1988; 52: 30-41.

18 Meerkerk GJ, Van Den Eijnden RJ, Vermulst AA, Garretsen HF. The Compulsive Internet Use Scale (CIUS): some psychometric properties. Cyberpsychol Behav 2009; 12(1): 1-6.

19 Biederman J, Newcorn J, Sprich S. Comorbidity of attention deficit hyperactivity disorder with conduct, depressive, anxiety, and other disorders. Am J Psychiatry 1991; 148: 564-77.

20 Wechsler D. Wechsler Preschool and Primary Scale of Intelligence-Revised. The Psychological Corporation, 1990.
21 Achenbach TM. Manual for the Child Behavior Checklist/4-18 and 1991 Profile. University of Vermont, 1991.

22 StataCorp. Stata Statistical Software: Release 14. StataCorp, 2015 (https:/ www.stata.com/stata14/)

23 Birnbaum HG, Kessler RC, Lowe SW, Secnik K, Greenberg PE, Leong SA, et al. Costs of attention deficit-hyperactivity disorder (ADHD) in the US: excess costs of persons with ADHD and their family members in 2000. Curr Med Res Opin 2005; 21(2): 195-205.

24 Pingault JB, Tremblay RE, Vitaro F, Carbonneau R, Genolini C, Falissard B, et al. Childhood trajectories of inattention and hyperactivity and prediction of educational attainment in early adulthood: a 16-year longitudinal population-based study. Am J Psychiatry 2011; 168: 1164-70.

25 Cortese S, Moreira-Maia CR, St Fleur D, Morcillo-Peñalver C, Rohde LA Faraone SV. Association between ADHD and obesity: a systematic review and meta-analysis. Am J Psychiatry 2016; 173(1): 34-43.

26 Demontis D, Walters RK, Martin J, Mattheisen M, Als TD, Agerbo E, et al. Discovery of the first genome-wide significant risk loci for ADHD. bioRxiv 2018; doi: 10.1101/145581.

27 Klein RG, Mannuzza S, Ramos Olazagasti MA, Roizen E, Hutchison JA Lashua EC, et al. Clinical and functional outcome of childhood attention-deficit/ hyperactivity disorder 33 years later. Arch Gen Psychiatry 2012; 69(12): 1295303.

28 Hechtman L, Swanson JM, Sibley MH, Stehli A, Owens EB, Mitchell JT, et al. Functional adult outcomes 16 years after childhood diagnosis of attentiondeficit/hyperactivity disorder: MTA results. J Am Acad Child Adol Psychiatry 2016: 55(11): $945-52$

29 Swanson EN, Owens EB, Hinshaw SP. Pathways to self-harmful behaviors in young women with and without ADHD: a longitudinal examination of mediating factors. J Child Psychol Psychiatr 2014; 55(5): 505-15.

30 Ustun B, Adler LA, Rudin C, Faraone SV, Spencer TJ, Berglund P, et al. The World Health Organization adult attention-deficit/hyperactivity disorder self-report screening scale for DSM-5. JAMA Psychiatry 2017; 74(5): 520-6.

31 Polanczyk G, de Lima MS, Horta BL, Biederman J, Rohde LA. The worldwide prevalence of ADHD: a systematic review and metaregression analysis. $A m$ J Psychiatry 2007; 164: 942-48.

32 Scahill L, Schwab-Stone M, Merikangas KR, Leckman JF, Zhang H, KasI S. Psychosocial and clinical correlates of ADHD in a community sample of schoolage children. J Am Acad Child Adol Psychiatr 1991; 38(8): 976-84.

\section{psychiatry in literature}

\section{On Worry}

\section{Aruna Rose Mary Kapanee}

Bhartrihari an Indian philosopher, poet and grammarian who lived around the fifth century CE and wrote in the Old Indo-Aryan language of Sanskrit, compared the words 'chinta' meaning 'worry, anxiety', with 'chita' meaning 'funeral pyre'. 'Chinta' is distinct from its corresponding word 'chintana', which means 'thinking, reflecting'. 'Chita' is made of wood and is used in traditional Hindu rites to burn dead bodies. When Sanskrit is written in the Devanagari script, the spelling of the two words 'chinta' and 'chita' is similar except for an 'anusvara', which is a single dot above a letter, that differentiates the two words. Bhartrihari’' literary works included 'Subhashita', which literally means 'good speech', and are Sanskrit epigrammatic verse that convey wisdom and instruction. In one of his 'Subhashitas', he writes that whereas the difference between the words 'chinta' and 'chita' lies in a dot; the funeral pyre sets ablaze the dead, while worry burns and renders lifeless the living. In a similar vein, pathological worry has been indicated through research to be associated with a range of mental disorders including - anxiety, eating and alcohol use-disorders; as well as with an increased risk for developing certain physical illnesses such as gastrointestinal and heart disease. 